\title{
Comparative Study of Disease Suppression on Various Host Plants by Bacillus Cyclic Lipopeptides
}

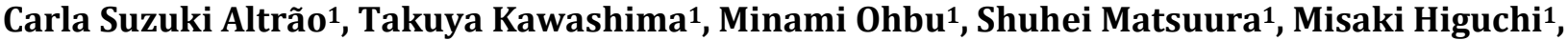 Yusuke Yanai', Yuki Hase1, Hirosuke Shinohara ${ }^{2}$, Kenji Yokota ${ }^{{ }^{*}}$}

${ }^{1}$ Department of Agricultural Chemistry, Tokyo University of Agriculture, Setagaya, Japan

${ }^{2}$ Department of Agriculture, Tokyo University of Agriculture, Atsugi, Japan

Email: *yokota@nodai.ac.jp

How to cite this paper: Altrão, C.S., Kawashima, T., Ohbu, M., Matsuura, S., Higuchi, M., Yanai, Y., Hase, Y., Shinohara, H. and Yokota, K. (2022) Comparative Study of Disease Suppression on Various Host Plants by Bacillus Cyclic Lipopeptides. Agricultural Sciences, 13, 1-9.

https://doi.org/10.4236/as.2022.131001

Received: December 14, 2021

Accepted: January 14, 2022

Published: January 17, 2022

Copyright $\odot 2022$ by author(s) and Scientific Research Publishing Inc. This work is licensed under the Creative Commons Attribution-NonCommercial International License (CC BY-NC 4.0). http://creativecommons.org/licenses/by-nc/4.0/

\begin{abstract}
In biological controls using Bacillus spp., cyclic lipopeptides play a role as elicitors to induce disease resistance on various host plants. However, it is still unclear the specificity between cyclic lipopeptides and host plants to induce disease resistance. In this study, we aimed to clarify the specificity to induce disease resistance among cyclic lipopeptides on various host plants. Our data clearly showed both cyclic lipopeptides conferred disease suppression on most of host plants, but at different range of cyclic lipopeptide concentration. Our findings contribute to understanding the complex on the specificity of cyclic lipopeptide derived induced disease resistance.
\end{abstract}

\section{Keywords}

Bacillus, Cyclic Lipopeptide, Induced Disease Resistance, Surfactin, Iturin

\section{Introduction}

Cyclic lipopeptides (cLPs) are well known to play key roles in biological controls against several kinds of plant diseases by using Bacillus spp. as biological control agents [1]. These molecules are produced through the non-ribosomal synthesis of peptides, coordinated by a complex of multienzymes (NRPSs) which catalyze all necessary steps including the ordered selection and condensation of amino acid residues [2]. So far, a variety of cLPs derived from Bacillus spp. have been identified and are categorized into 3 families: surfactin family, iturin family and fengycin family, depending on the chemical structure and biosynthetic genes [2].

Surfactin and iturin A are well studied compounds among the Bacillus cLPs. 
Surfactin A consists of a $\beta$-hydroxy fatty acid with a heptapeptide ELLVDLL, and iturin $\mathrm{A}$ consists of a $\beta$-amino fatty acid with a heptapeptide NYNQPNS. Both cLPs show antimicrobial activity [3] [4] [5].

cLPs also behave as elicitors to induce disease resistance on several kinds of plants. Rice [6], strawberry [7], and grapevine [8] have been reported to show disease suppression depending on the cLPs used to induce disease resistance. Although cLPs induce disease resistance on host plants with a broad host range, there is also some specificity among the combinations of cLP molecules and host plants. However, the factors that determine the abilitiy of cLPs to induce disease in plants and how they work from different hosts are still unclear.

We previous reported that both purified surfactin and iturin A show disease suppression against soil-borne diseases caused by Fusarium oxysporum on tatsoi and lettuce, respectively [9] [10]. However, excess amounts of cLP amendments in soil negate the disease suppression for both of surfactin and iturin A.

In this study, we aimed to clarify the effect of surfactin and iturin A to suppress disease via induced disease resistance in various edible plants.

\section{Materials and Methods}

\subsection{Plants and Microbes}

Plants and microbes used in this study are listed in Table 1.

Table 1. Plants and microbes used in this study.

\begin{tabular}{|c|c|c|}
\hline Plants & & \\
\hline Scientific name & Cultivar & Common name \\
\hline Brassica oleracea & Ranten (Sakata Seed, Kanagawa, Japan) & Cabbage \\
\hline Solanum lycopersicum & Frutica (Takii, Kyoto, Japan) & Tomato \\
\hline Oryza sativa & Nipponbare (Nouken, Kyoto, Japan) & Rice \\
\hline Glycine max & Okuharawase (Sakata Seed, Kanagawa, Japan) & Soybean \\
\hline Cucumis sativus & Sagami Hanjiro (Noguchi Seeds, Saitama, Japan) & Cucumber \\
\hline \multicolumn{3}{|l|}{ Microbes } \\
\hline Strain & Description & $\begin{array}{l}\text { Bacterial suspension for } \\
\text { inoculation (cells } \mathrm{mL}^{-1} \text { ) }\end{array}$ \\
\hline $\begin{array}{l}\text { Pseudomonas syringae pv. } \\
\text { maculicola MAFF } 302783\end{array}$ & Causal agent of bacterial leaf spot of cabbage & $10^{5}$ \\
\hline P. syringae pv. tomato MAFF 302665 & Causal agent of bacterial speck of tomato & $10^{3}$ \\
\hline P. syringae pv. oryzae MAFF 301529 & Causal agent of bacterial halo blight of rice & $10^{9}$ \\
\hline P. syringae pv. glycinea MAFF 301683 & Causal agent of bacterial blight of soybean & $10^{4}$ \\
\hline $\begin{array}{l}\text { P. syringae pv. lachrymans } \\
\text { MAFF } 301322\end{array}$ & Causal agent of marginal blight of cucumber & $10^{4}$ \\
\hline Bacillus subtilis ATCC 21556 & Iturin A-producing bacteria & \\
\hline
\end{tabular}




\subsection{Cyclic Lipopeptides}

Surfactin sodium salt was purchased from FUJIFILM Wako Pure Chemical Corporation (Osaka, Japan). Iturin A was purified from solid-state cultures of $B$. subtilis ATCC21556 by organic solvent extraction followed by column chromatography, as previously described [9]. Briefly, solid-state culture of B. subtilis ATCC 21556 was extracted by ethyl acetate (EtOAc): 1-butanol $(\mathrm{BuOH})=7: 3$ $(\mathrm{v} / \mathrm{v})$, and then, organic phase was dried up by a rotary evaporator. The resulting residue was dissolved in EtOAc: methanol $(\mathrm{MeOH})=90: 10(\mathrm{v} / \mathrm{v})$ and applied to a silica gel column (SI60 $\mu$ m SIZE:60., Shoko Science, Kanagawa, Japan). Iturin A was eluted by a linear gradient of EtOAc - $\mathrm{MeOH}$ (90:10 to 50:50 within $30 \mathrm{~min}$ ). The elution containing iturin A were combined and dried up by a rotary evaporator. The resulting residue was dissolved in $50 \% \mathrm{MeOH}$, and applied to an ODS column (Octa Decyl Silyl) resin (Wakogel 50C18, Wako Pure Chemical Industries, Osaka, Japan). Iturin A was eluted by a linear gradient of $\mathrm{MeOH}$ - DDW (50:50 to 75:25 within $60 \mathrm{~min}$, followed by 75:25 for $30 \mathrm{~min}$ ). Purified Iturin A was $>98 \%$ pure measured at $210 \mathrm{~nm}$ by HPLC.

\subsection{Disease Suppression Assay}

Disease suppression assay was conducted in an air-controlled greenhouse at $24^{\circ} \mathrm{C}$. Host plants were propagated by a hydroponic culture with Hoagland solution [11] unless otherwise described. Population of bacterial suspensions for inoculation on host plants is listed in Table 1.

\subsubsection{Rice}

Rice plants were cultivated with Kasugai solution [12]. At the seven-leaf stage, purified surfactin or iturin A was added to hydroponic solution for root-treatment on host plants.

Pseudomonas syringae pv. oryzae MAFF 301529, the causal agent of bacterial halo blight on rice, was grown on $\mathrm{LB}$ agar plate at $25^{\circ} \mathrm{C}$ for 2 days. The bacterial colony was obtained and suspended in sterilized $0.1 \% \mathrm{w} / \mathrm{v}$ Tween 20 solution. Five microliters of bacterial suspension was inoculated on true leaves by puncturing method. After inoculation of pathogen, rice plantlets were covered by plastic bags for $24 \mathrm{~h}$ in the greenhouse to maintain high humidity. Disease severity was rated using a 0 to 3 rating scale ( 0 , no disease symptom; 1 , pale green or pale yellowish symptoms; 2, yellow or yellowish-brown symptoms; 3 , conspicuous halo symptom) [13].

\subsubsection{Other Plants}

Bacterial pathogens (Table 1) were grown on King's B agar plate at $25^{\circ} \mathrm{C}$ for 2 days. Bacterial colonies were obtained and suspended in sterilized $10 \mathrm{mM} \mathrm{MgSO}_{4}$ solution. The bacterial suspension was inoculated by infiltration on the abaxial side of leaves. After inoculation of pathogen, plantlets were covered by plastic bags for $24 \mathrm{~h}$ in the green house to keep high humidity. Disease incidence was expressed in terms of the percentage of disease symptoms. 


\subsubsection{Statistical Analysis}

All statistical analysis was achieved using R v.3.5.1 (https://www.r-project.org/). Disease severity on rice was performed by Wilcoxon's U-test. Disease incidence on other host plants was performed by Student's T-test.

\section{Results and Discussion}

\subsection{Cabbage}

We previously reported on disease suppression by soil amendments of purified surfactin or iturin A against Fusarium yellows of tatsoi, Brassica rapa var. rosularis [9]. We found that both surfactin and iturin A significantly suppress disease caused by this soil-borne pathogen. However, the disease suppression by surfactin and iturin A can be negated by applying excess amounts of either cLP in soil.

Surfactin is a strong haemolytic, antibacterial and antiviral molecule [14]. By contrast, members of iturin family present limited antibacterial and no antiviral activity but they have a strong in vitro antifungal action [15]. Beyond that, these molecules have been reported as inducers of defense response in host plant by stimulation of the induced systemic resistance phenomenon [7] [16].

Significant disease suppression against bacterial cabbage leaf spot was observed after root-treatment of host plants with both surfactin and iturin $\mathrm{A}$ at a range of 0.125 to $1 \mu \mathrm{M}$ (Figure 1(a) and Figure 1(b)). Moreover, disease suppression was negated after application of more than $2 \mu \mathrm{M}$ of root-treatments for both of surfactin and iturin $\mathrm{A}$, respectively, whereas no disease symptoms were observed with $4 \mu \mathrm{M}$ of surfactin or iturin A root-treatments without pathogen-inoculation (Figure 1(a) and Figure 1(b)).

This data more clearly demonstrates that the disease suppression is due to the effects of induced disease resistance by surfactin and iturin A, instead to the antimicrobial activities of surfactin and iturin A directly against soil-borne pathogens. Furthermore, it is notable that the loss of disease suppressive activities of surfactin and iturin A at high concentrations occurred via lack of induced disease resistance, with no visible plant disease symptoms observed unless a pathogen is applied.

\subsection{Tomato}

On tomato, significant disease suppression against bacterial speck was observed by root-treatment of surfactin at a range of 1 to $4 \mu \mathrm{M}$ (Figure 1(c) and Figure $1(d)$ ). On the other hand, no significant disease suppression was observed at a range of 0.25 to $32 \mu \mathrm{M}$ of iturin A treatments.

In tobacco (Nicotiana tabacum), which belongs to the same family (Solanaceae) as tomato, surfactin treatment of tobacco cells elicits induced disease resistance related events, whereas no responses were observed following iturin A treatments [17]. Our data suggests that a specificity between surfactin and Solanaceae plants may exist for eliciting induced disease resistance.

Similar to cabbage, high surfactin treatments over $8 \mu \mathrm{M}$ negated disease 

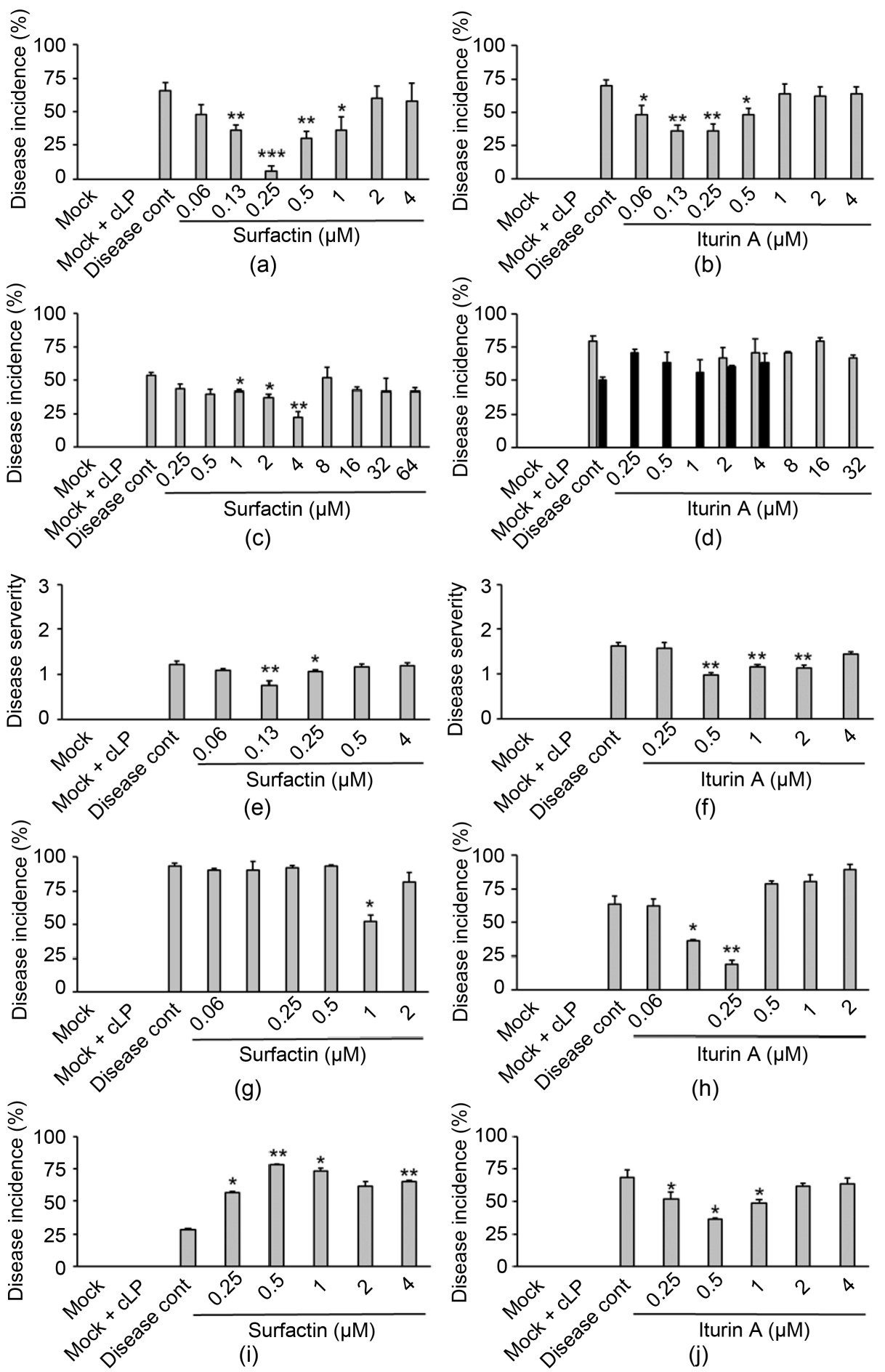

Figure 1. Disease suppression following root treatment of surfactin or iturin A against bacterial leaf disease on various kinds of host plants. (a) surfactin-treatment on cabbage $(n=5),(b)$ iturin A-treatment on cabbage $(n=5)$, (c) surfactin-treatment on tomato $(\mathrm{n}=3)$, (d) iturin A-treatment on tomato $(\mathrm{n}=3)$, (e) surfactin-treatment on rice $(\mathrm{n}=3)$, (f) iturin A-treatment on rice $(\mathrm{n}=$ 3), (g) surfactin-treatment on soybean $(\mathrm{n}=3)$, (h) iturin A-treatment on soybean $(\mathrm{n}=3)$, (i) surfactin-treatment on cucumber $(\mathrm{n}=$ 3), (j) iturin A-treatment on cucumber $(n=3)$. Mean \pm SE of disease incidence or disease severity are represented. Asterisks indicate significant difference from disease control: ${ }^{\star}, P<0.05$; ${ }^{\star *}, P<0.01$. The concentrations of cLP in Mock + cLP were set as the highest concentration for each experiment. Two independent experiments testing a range of concentrations of iturin A-treatments are represented by gray and black bars in panel $\mathrm{D}$. 
suppression activities whereas no disease symptoms were observed with up to 32 $\mu \mathrm{M}$ of surfactin treatment without pathogen inoculation.

\subsection{Rice}

On rice, both surfactin and iturin A showed disease suppression activity against bacterial leaf blight of rice after root treatments. However, the range of concentrations that elicited disease suppression differed between surfactin and iturin $\mathrm{A}$ (Figure 1(e) and Figure 1(f)). The range of concentration that showed significant disease suppression by surfactin was 0.06 to $0.5 \mu \mathrm{M}$ in hydroponic culture. This disease suppression range was lower than that observed for iturin A treatments, which was 0.5 to $2 \mu \mathrm{M}$ in hydroponic culture. Moreover, similar to the above plants, $1 \mu \mathrm{M}$ of surfactin or $4 \mu \mathrm{M}$ of iturin $\mathrm{A}$ treatments negated the observed disease suppression, whereas no disease symptoms were observed at $1 \mu \mathrm{M}$ of surfactin- and $4 \mu \mathrm{M}$ of iturin A-treatments, respectively, in pathogen-free plants.

On disease suppression through treatment of purified surfactin or mycosubtilin, a member of iturin family, against rice sheath blight on rice, and followed the rice plant response through gene expression analysis for induced disease resistance related genes [6]. With respect to their findings, both surfactin and mycosubtilin elicited expression of induced disease resistance related genes on rice. However, in that study, only surfactin showed disease suppressive activity at the $35 \mu \mathrm{M}$ treatment used against the fungal disease on rice, whereas no disease suppression was observed following mycosubtilin treatment at the same concentration. Based on our findings, we propose that mycosubtilin might also show disease suppression against rice fungal disease at a different range of mycosubtilin concentration.

\subsection{Soybean}

On soybean, both purified surfactin and iturin A treatments showed significant disease suppression against bacterial blight of soybean (Figure 1(g) and Figure 1(h)). However, the concentration of surfactin that showed significant disease suppression was $1 \mu \mathrm{M}$ in hydroponic culture, which was higher than the significantly effective range of iturin A treatment $(0.13$ and $0.25 \mu \mathrm{M}$ in hydroponic culture). Moreover, $2 \mu \mathrm{M}$ of surfactin or $0.5 \mu \mathrm{M}$ of iturin A treatments negated the disease suppression, whereas no disease symptoms were observed by both surfactin- and iturin A-treatments at $2 \mu \mathrm{M}$ in pathogen-free soybean plants.

\subsection{Cucumber}

The treatment of cucumber seeds with a mixture of $1 \mu \mathrm{M}$ of semi-purified of iturin, surfactin and fengycin, respectively, showed no disease suppression against cucurbit anthracnose on cucumber leaf caused by Colletotrichum lagenarium [18].

In this study, iturin A treatments of cucumber roots showed significant disease suppression against cucurbit angular leaf spot at a range of 0.25 to $1.0 \mu \mathrm{M}$ in 
hydroponic culture, whereas the negation of disease suppression was observed at $2 \mu \mathrm{M}$ and higher of iturin A in hydroponic culture (Figure 1(i) and Figure 1(j)), similar to the other plants above. On the other hand, surprisingly, significant disease enhancement was observed following surfactin treatments at a range of 0.25 to $4 \mu \mathrm{M}$ at 3 days post inoculation. With respect to the previous report [15], we deduce that by including surfactin in the mixture of cLPs, it might have acted antagonistically against the disease suppression by iturin $\mathrm{A}$.

\section{Conclusions}

In this present study, we investigated that disease suppression elicited by purified surfactin- and iturin A-treatments against bacterial leaf diseases on various kinds of edible plants. The induce resistance promoted by cLPs required different concentration range of iturin A or surfactin depending on host plant. Based on our observations, most of the studied host plants were elicited by cLP treatment on roots to suppress diseases through induced disease resistance, and the effective disease-suppressing concentrations varied by host and cLP.

The negation of disease suppressive activity that was observed at excess concentrations of either surfactin or iturin A for all host plants was confirmed to be through loss of disease suppression. It is still unclear how host plants recognize cLPs. Further study should be done by analyzing gene expression of induced disease resistance related genes at a variety of cLP concentrations.

\section{Acknowledgements}

We thank Dr. Adrien Burch for English correction and discussion. We thank Mr. Shohei Fujita in Sakata seeds for kindly providing seeds of cabbage. This work is supported by JSPS KAKENHI Grant Number JP16K07628, and Tokyo NODAI Doctoral Research Grant Program 46407328F and MEXT (Ministry of education, culture, sports, science and technology Japan).

\section{Conflicts of Interest}

The authors declare no conflicts of interest regarding the publication of this paper.

\section{References}

[1] Romero, D., De Vicente, A., Olmos, J.L., Dávila, J.C. and Pérez-García, A. (2007) Effect of Lipopeptides of Antagonistic Strains of Bacillus subtilis on the Morphology and Ultrastructure of the Cucurbit Fungal Pathogen Podosphaera fusca. Journal of Applied Microbiology, 103, 969-976. https://doi.org/10.1111/j.1365-2672.2007.03323.x

[2] Stein, T. (2005) Bacillus subtilis Antibiotics: Structures, Syntheses and Specific Functions. Molecular Microbiology, 56, 845-857. https://doi.org/10.1111/j.1365-2958.2005.04587.x

[3] Peypoux, F., Guinand, M., Michel, G., Delcambe, L., Das, B.C. and Lederer, E. (1978) Structure of Iturine A, a Peptidolipid Antibiotic from Bacillus subtilis. Bio- 
chemistry, 17, 3992-3996. https://doi.org/10.1021/bi00612a018

[4] Arima, K., Kakinuma, A. and Tamura, G. (1968) Surfactin, a Crystalline Peptidelipid Surfactant Produced by Bacillus subtilis. Isolation, Characterization and Its Inhibition of Fibrin Clot Formation. Biochemical and Biophysical Research Communications, 31, 488-494. https://doi.org/10.1016/0006-291X(68)90503-2

[5] Kakinuma, A., Sugino, H., Isono, M., Tamura, G. and Arima, K. (1969) Determination of Fatty Acid in Surfactin and Elucidation of the Total Structure of Surfactin. Agricultural and Biological Chemistry, 33, 973-976. https://doi.org/10.1080/00021369.1969.10859409

[6] Chandler, S., Van Hese, N., Coutte, F., Jacques, P., Höfte, M. and De Vleesschauwer, D. (2015) Role of Cyclic Lipopeptides Produced by Bacillus subtilis in Mounting Induced Immunity in Rice (Oryza sativa L.). Physiological and Molecular Plant Pathology, 91, 20-30. https://doi.org/10.1016/j.pmpp.2015.05.010

[7] Yamamoto, S., Shiraishi, S. and Suzuki, S. (2015) Are Cyclic Lipopeptides Produced by Bacillus amyloliquefaciens S13-3 Responsible for the Plant Defence Response in Strawberry against Colletotrichum gloeosporioides? Letters in Applied Microbiology, 60, 379-386.

[8] Farace, G., Fernandez, O., Jacquens, L., Coutte, F., Krier, F., Jacques, P., Clément, C., Barka, E.A., Jacquard, C. and Dorey S. (2015) Cyclic Lipopeptides from Bacillus subtilis Activate Distinct Patterns of Defence Responses in Grapevine. Molecular Plant Pathology, 16, 177-187.

[9] Yokota, K. and Hayakawa, H. (2015) Impact of Antimicrobial Lipopeptides from Bacillus sp. on Suppression of Fusarium Yellows of Tatsoi. Microbes and Environments, 30, 281-283. https://doi.org/10.1264/jsme2.ME15062

[10] Fujita, S. and Yokota, K. (2019) Disease Suppression by the Cyclic Lipopeptides Iturin A and Surfactin from Bacillus spp. against Fusarium Wilt of Lettuce. Journal of General Plant Pathology, 85, 44-48. https://doi.org/10.1007/s10327-018-0816-1

[11] Hoagland, D.R. and Arnon, D.I. (1950) The Water-Culture Method for Growing Plants without Soil. California Agricultural Experiment Station, Circular-347.

[12] Kasugai, S. (1939) Studies on the Hydroponic Cultures. Journal of the Science of Soil and Manure, 13, 669-822.

[13] Kuwata, H. (1985) Pseudomonas syringae pv. oryzae pv. nov., Causal Agent of Bacterial Halo Blight of Rice. Japanese Journal of Phytopathology, 51, 212-218. https://doi.org/10.3186/jiphytopath.51.212

[14] Ongena, M. and Jacques, P. (2008) Bacillus Lipopeptides: Versatile Weapons for Plant Disease Biocontrol. Trends in Microbiology, 16, 115-125. https://doi.org/10.1016/j.tim.2007.12.009

[15] Phae, C.G., Shoda, M. and Kubota, H. (1990) Suppressive Effect of Bacillus subtilis and It's Products on Phytopathogenic Microorganisms. Journal of Fermentation and Bioengineering, 69, 1-7. https://doi.org/10.1016/0922-338X(90)90155-P

[16] Ongena, M., Jourdan, E., Adam, A., Paquot, M., Brans, A., Joris, B., Arpigny, J.L., Paquot, M. and Thonart, P. (2007) Surfactin and Fengycin Lipopeptides of Bacillus subtilis as Elicitors of Induced Systemic Resistance in Plants. Environmental Microbiology, 9, 1084-1090. https://doi.org/10.1111/j.1462-2920.2006.01202.x

[17] Jourdan, E., Henry, G., Duby, F., Dommes, J., Barthélemy, J.P., Thonart, P. and Ongena M. (2009) Insights into the Defense-Related Events Occurring in Plant Cells Following Perception of Surfactin-Type Lipopeptide from Bacillus subtilis. Molecular Plant-Microbe Interactions, 22, 456-468. 
[18] Ongena, M., Duby, F., Jourdan, E., Beaudry, T., Jadin, V., Dommes, J. and Thonart, P. (2005) Bacillus subtilis M4 Decreases Plant Susceptibility towards Fungal Pathogens by Increasing Host Resistance Associated with Differential Gene Expression. Applied Microbiology and Biotechnology, 67, 692-698. 